\title{
Scanning Transmission Electron Tomography
}

\author{
P.A. Midgley, ${ }^{*}$ M. Gass, ${ }^{*}$ I. Arslan, ${ }^{* *}$ J. Tong, ${ }^{*}$ T.J.V. Yates, ${ }^{*}$ A. Hungria, ${ }^{*}$ R.E. Dunin-
} Borkowski, $*$ M. Weyland *** and J.M. Thomas*

* Department of Materials Science and Metallurgy, University of Cambridge, Pembroke Street, Cambridge, CB2 3QZ, United Kingdom.

** Micro and Interfacial Sciences Department, Sandia National Laboratories, 7011 East Avenue, Livermore CA 94550, USA.

*** E13 Clark Hall, Department of Applied and Engineering Physics, Cornell University, Ithaca, NY 14853, USA.

Whilst electron tomography has been used in various forms in the life sciences for over thirty years, it is only in the last 5 years or so that electron tomography has been adopted in the materials sciences as a tool of importance. In particular the desire to study the three dimensional structure, composition and physical properties of devices and materials at the nanoscale has driven the development of new forms of electron tomography, for example combining energy filtered TEM, electron holography and weak beam dark field imaging with tomographic techniques to elucidate features unobtainable with conventional 2D imaging. However, by far the most commonly used mode of electron tomography in materials science is that of scanning transmission electron tomography predominantly using STEM high angle annular dark field (HAADF) imaging as a signal for tomographic analysis. The predominance of STEM-based tomography arises from two factors; firstly the incoherent nature of the HAADF signal which minimizes the effects of diffraction contrast, anathema to successful tomographic reconstruction, and secondly the high contrast afforded by the dependence of the scattered intensity to the atomic number, $Z$ (approximately $Z^{2}$ ). This makes the STEM signal ideal for materials tomography and as such it has become the conventional imaging mode for three dimensional studies at the nanoscale in materials science [1].

Here we present some recent examples which illustrate the power of STEM based tomography for crystallographic and metrological studies. A series of STEM HAADF images were recorded of a small ( 100nm diameter) fragment of mesoporous silica MCM 48, a three dimensional porous structure exhibiting a double gyroid structure with space group $I a \overline{3} d$. The images were recorded about a single tilt axis whose direction was not known before acquisition. After reconstruction the crystal was aligned and slices taken perpendicular to major zone axis. Fig. 1(a) shows a reconstructed slice perpendicular to a $<110>$ direction. A Bragg-filtered version, used to reduce nonperiodic artefacts, has been superimposed for clarity. The inset shows a power spectrum of the slice indicating strong pairs of Fourier components, which if indexed according to a $<110>$ diffraction pattern, correspond to $\{332\}$ and $\{224\}$ reflections, the latter set demonstrating $4.7 \mathrm{~nm}$ periodic resolution within the slice. Fig. 1(b) shows a projection parallel to a boundary separating two parts of the fragment separated by a rotation about a common $<112>$ axis [2].

Magneto-tactic bacteria are composed of a backbone of iron oxide or iron sulphide crystals which is used by the bacterium to sense the earth's geomagnetic field. STEM HAADF images of such bacteria were used to enable a reconstruction of the crystallites to be made with sufficient clarity that measurements of the facet size could be used to elucidate crystal growth trends. Fig. 2 (a) shows a 
typical reconstructed crystal with parameters indicated, subsequently used to emphasize a growth trend from immature to mature crystals, see Fig. 2 (b) [3].

\section{References}

[1] P.A. Midgley and M. Weyland Ultramicroscopy 96 (2003) 413.

[2] T.J.V. Yates et al., Chem. Phys Lett. 418 (2006) 540.

[3] This research was supported by the EPSRC, the Isaac Newton Trust, FEI Company, the Royal Academy of Engineering and the Leverhulme Trust.
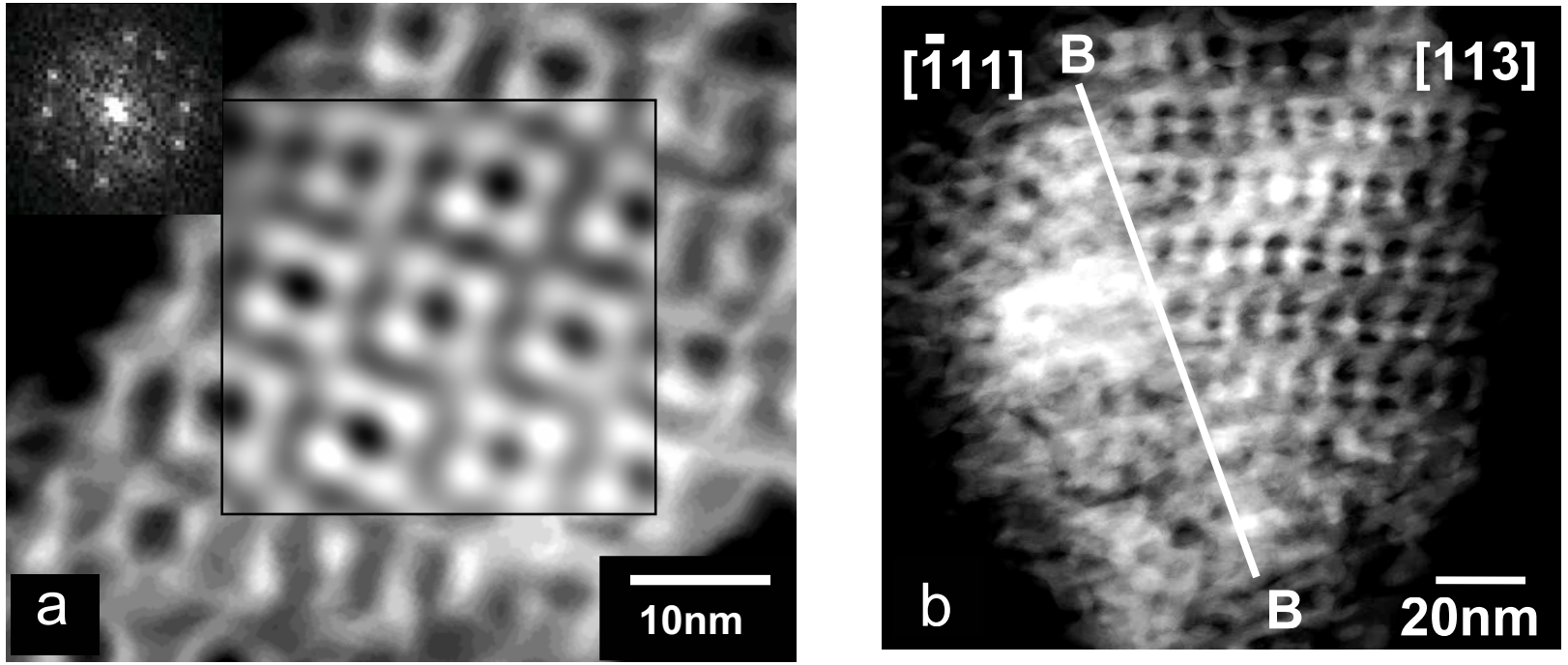

FIG. 1. (a) A reconstructed slice through a reconstruction of the mesoporous silica MCM-48, perpendicular to the $\langle 110\rangle$ zone axis. A Bragg-filtered inset enhanced the periodic detail. The pairs of spots in the power spectrum correspond to $\{332\}$ and $\{224\}$ reflections. (b) A view of a boundary (BB) separating two 'grains' of MCM-48 with [113] orientation (right) and [1 11] orientation (left).
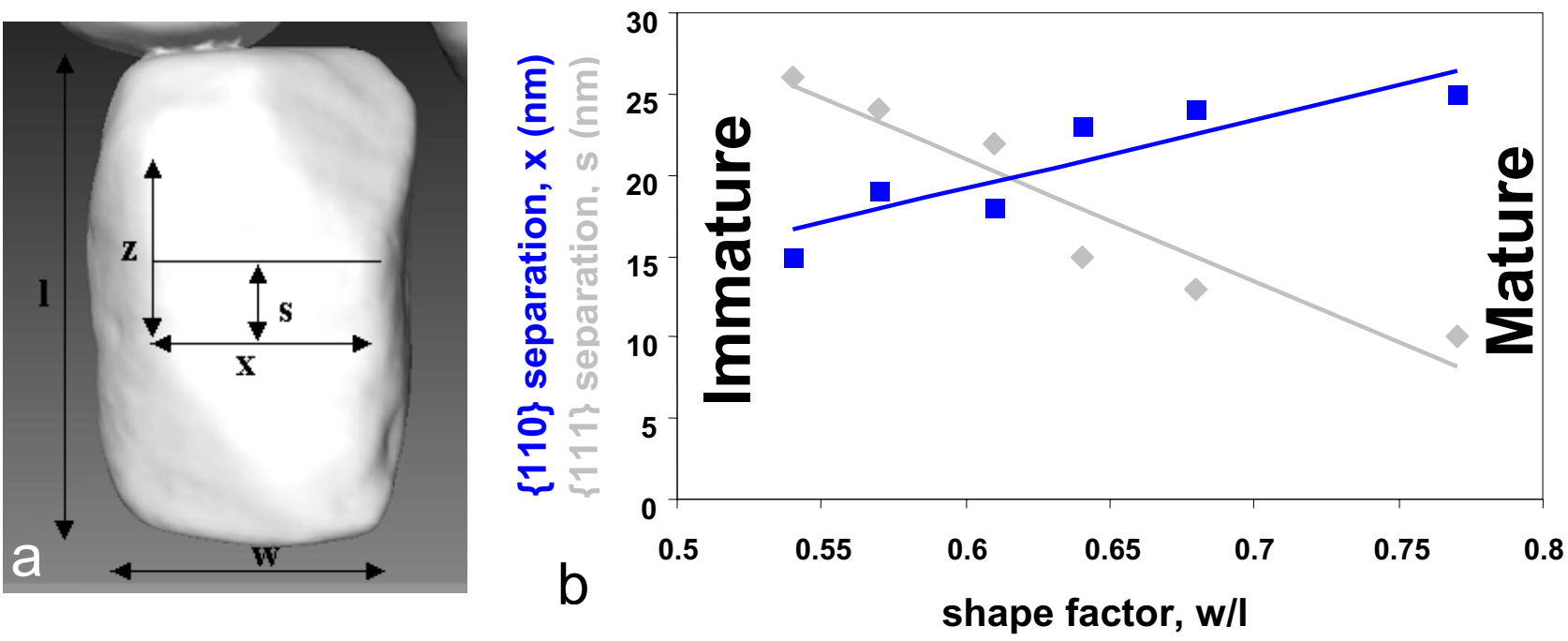

FIG. 2. (a) A surface render of a magnetotactic bacterium crystal (magnetite) with measured parameters used to determine a growth trend (b) indicating the increase in shape factor as the crystal matures. 